\title{
Acknowledgment to Reviewers
}

(c) The Phytopathological Society of Japan and Springer Japan KK, part of Springer Nature 2021

\author{
Yuki Ichinose \\ Editor-in-Chief, \\ Journal of General Plant Pathology
}

The success of the Journal of General Plant Pathology largely depends on the quality of manuscripts submitted by authors, on the careful review by associate editors, and on the painstaking proofreading by editorial managers. In addition to the associate editors and the editorial managers, the reviewers listed below provided constructive critical reviews of at least one manuscript during the year 2021. Their names are published here in grateful appreciation for their significant contribution to the journal as well as to the development of plant pathology.

$\begin{array}{lll}\text { Seishi AKINO } & \text { Yasuhiro INOUE } & \text { Tomoo MISAWA } \\ \text { Nicolas BEJERMAN } & \text { Hiroki IRIEDA } & \text { Susumu MOCHIZUKI } \\ \text { Ali Chenari BOUKET } & \text { Yasuhiro ISHIGA } & \text { Jouji MORIWAKI } \\ \text { Sotaro CHIBA } & \text { Kazuya ISHIKAWA } & \text { Keiichi MOTOHASHI } \\ \text { Mohsen ELSHARLAWY } & \text { Shin-ichi ITO } & \text { Takafumi MUKAIHARA } \\ \text { Shin-ichi FUJI } & \text { Yoko ITO } & \text { Jiro MURAKAMI } \\ \text { Takashi FUJIKAWA } & \text { Kosuke IZUMITSU } & \text { Kiichi NAKAYAMA } \\ \text { Kohki FUJISAKI } & \text { Yasuhiro KADOTA } & \text { Yutaro NERIYA } \\ \text { Fumi FUKADA } & \text { Shigeyuki KAKIZAWA } & \text { Takamichi NIJO } \\ \text { Ayako FURUTANI } & \text { Satoko KANEMATSU } & \text { Hisashi NISHIGAWA } \\ \text { Kenji GOMI } & \text { Takeshi KANTO } & \text { Kouhei OHNISHI } \\ \text { Shu HASE } & \text { Shinpei KATOU } & \text { Kazusato OHSHIMA } \\ \text { Masayoshi HASHIMOTO } & \text { Miyuki KAYAMORI } & \text { Yukari OKANO } \\ \text { Ayaka HIENO } & \text { Kappei KOBAYASHI } & \text { Kenro OSHIMA } \\ \text { Hisae HIRATA } & \text { Ken KOMATSU } & \text { Hiromasa SAITOH } \\ \text { Kazuyuki HIRATSUKA } & \text { Norio KONDO } & \text { Atsuko SASAKI } \\ \text { Yuuri HIROOKA } & \text { Toru KONDO } & \text { Hiroyuki SAWADA } \\ \text { Kiwamu HYODO } & \text { Kenji KUBOTA } & \text { Shigemi SEO } \\ \text { Tamaki UEHARA-ICHIK } & \text { Masaharu KUBOTA } & \text { Masahiro SHISHIDO } \\ \text { Yuichiro IIDA } & \text { Kensaku MAEJIMA } & \text { Nobutaka SOMEYA } \\ \text { Kazuhiro IIYAMA } & \text { Malek MARIAN } & \text { Haruhisa SUGA } \\ \text { Kentaro IKEDA } & \text { Hayato MASUYA } & \text { Daigo TAKEMOTO } \\ \text { Iori IMAZAKI } & \text { Naoyuki MATSUMOTO } & \text { Minoru TAKESHITA } \\ & & \end{array}$

Yuichi TAKIKAWA

Kazuaki TANAKA

Nobuya TASHIRO

Takeshi TODA

Keisuke TOMIOKA

Elbeanio TOUFIC

Mizue TSUJI

Takao TSUKIBOSH

Shigenori UEDA

Toshiyuki USAMI

Shihomi UZUHASHI

Takashi YAENO

Kengo YAMADA

Kohji YAMAMURA

Nobuyuki YOSHIKAWA

Hirofumi YOSHIOKA

Chen YUH-KUN

Hideki WATANABE

Kyoko WATANABE

Xuehong WU

Publisher's Note Springer Nature remains neutral with regard to jurisdictional claims in published maps and institutional affiliations. 